\title{
Outcome of post caesarian pregnancy in a tertiary care hospital in Udaipur, Rajasthan
}

\author{
Archana Sharma, Harshdeep Jadeja, Preeti Malhotra*
}

Department of Obstetrics and Gynecology, Pacific Institute of Medical Sciences, Udaipur Rajasthan, India

Received: 29 January 2021

Revised: 11 March 2021

Accepted: 12 March 2021

\author{
*Correspondence: \\ Dr. Preeti Malhotra, \\ E-mail: drpreetimalhotra777@gmail.com
}

Copyright: (C) the author(s), publisher and licensee Medip Academy. This is an open-access article distributed under the terms of the Creative Commons Attribution Non-Commercial License, which permits unrestricted non-commercial use, distribution, and reproduction in any medium, provided the original work is properly cited.

\begin{abstract}
Background: The prevalence of caesarean section has increased due to a variety of factors such as patient choice and relative safety of the procedure. However in many cases the risk involved in LSCS is still present.

Methods: The present study was designed to evaluate the outcome in cases where VBAC or TOLAC were used for the second pregnancy in a previous LSCS case. The study employed a sample of 60 subjects who were equally divided in two groups who were subjected to elective cesarian and TOLAC.

Results: The results showed that TOLAC did have a better outcome as compared to repeat cesarian in context of maternal and foetal factors.

Conclusions: The practice of TOLAC must be tried as an alternative and can be a viable option for uncomplicated post cesarian deliveries.
\end{abstract}

Keywords: Cesarian section, TOLAC, VBAC

\section{INTRODUCTION}

Caesarean sections have become a cornerstone in a wide variety of obstetric centers due to the ease of procedure, swift completion and reduction in complications. The procedure has also been mandated to become one of the most recognizable procedures among non-medical individuals. Many women have reportedly been pursuing the elective route of caesarean section as it provides them with a relatively painless delivery while providing lower complications. ${ }^{1}$ However the use of caesarean is not without its disadvantages. The risk of post-operative complications and laxity on part of the mother or attendants in care can cause grave reactions. The cost of caesarean section in a non-government set up is another factor. There have been instances wherein studies have demonstrated an evidence-based conclusion of reduced maternal mortality in cases of vaginal birth after caesarean (VBAC). ${ }^{2,3}$ This conclusion has been accepted in majority of western as well as some Asian studies but evidence still needs to be produced in Indian scenarios.

It needs to be noted that choice for a caesarean section is not always an elective one and in instances of emergency $\mathrm{C}$ sections, the indications need to be noted. When opting for a VBAC, there must initially be a carefully drafted trial of labor after caesarean (TOLAC) to assess if the expectant mother is suitable and fit to undergo VBAC. The strategies and guidelines for TOLAC must be adhered to and should be reviewed periodically to establish a protocol that can be followed to obtain an optimum result in VBAC or to shift for a repeat caesarean. There are evidence in studies that mothers who undergo TOLAC have a $60-70 \%$ positive outcome for delivering vaginally thus reducing complications and reducing maternal morbidity. ${ }^{4,5}$ The present study was conducted to ascertain if VBAC provides a better 
maternal and neonatal outcome as compared to repeat caesarean section cases in the selected study sample.

\section{METHODS}

The study was a prospective study carried out in the department of obstetrics and gynecology at Pacific Institute of Medical Sciences, Udaipur Rajasthan over a period of one year from January 2019 to January 2020. The study involved expectant mothers who were regularly undergoing ANC in our institution and were admitted for delivery. The inclusion criteria was mother admitted for delivery with single previous caesarian section, and presently carrying a single foetus, in cephalic presentations and mothers pelvic anatomy was adequate for vaginal delivery. Mothers who had more than one $\mathrm{c}$ section, had a classical $\mathrm{C}$ section scar, malpresented foetal position were excluded. We also excluded all mothers who had pregnancy related disorders such as GDM, PIH etc.

The mothers and one attendant were counselled regarding the study and after obtaining written informed consent, they were included in the study cohort. The patients were asked to detail whether they preferred VBAC or elective repeat cesarian delivery, so that they could be included in the respective study group. Institutional ethical clearance was obtained prior to initiation of the study.

The study pool comprised of 60 subjects who were divided in two groups of 30 subjects each. The first group comprised of mothers who elected to undergo TOLAC, while the second comprised of subjects who opted for elective repeat cesarian. All TOLAC group members had the option to undergo cesarian section at any stage of time. The elective group were posted for $\mathrm{C}$ section after 39 weeks of delivery, while the TOLAC group was observed for spontaneous labor induction till 39 weeks after which induction was performed. All subjects were kept under monitoring for maternal and fetal parameters and were posted for $\mathrm{C}$ section if any indication arose.

Data analysis was done using SPSS software in consultation with institutional statistician. The data was subjected to tests for central tendency and associations.

\section{RESULTS}

The study involved 60 subjects among which 30 were posted for elective repeat cesarian section. Among the 30 subjects ready to undergo TOLAC, 14 (46.7\%) subjects had a VBAC, while 16 were posted for emergency LSCS. The commonest maternal complications that were seen in TOLAC group were scar dehiscence $(n=4,13.34 \%)$; followed by uterine rupture and infection. Although postoperative infection was seen higher in elective c section group $(n=8,26.67 \%)$ as compared to TOLAC group. Statistical analysis revealed that there was no significant difference in any of the complications except infection $(\mathrm{p}<0.05)$ among both the groups at $95 \%$ confidence interval.

In terms of neonatal outcome, APGAR score assessment done at end of 5 minutes revealed that only one neonate $(3.33 \%)$ in TOLAC group was having a score less than 7 , while $3(10 \%)$ neonates in elective caesarean group had a APGAR score less than 7 in the corresponding time period. Among the children delivered via VBAC, only 2 $(6.67 \%)$ required NICU care including pre term while in case of elective caesarean section, $8(26.67 \%)$ subjects required NICU admissions. In terms of neonatal complications, respiratory distress was observed in 2 cases, while elective group had 4 cases of respiratory depression. The data has been displayed in Fig 01 . Statistical analysis revealed that a significant difference existed between the neonatal outcomes between the two groups.

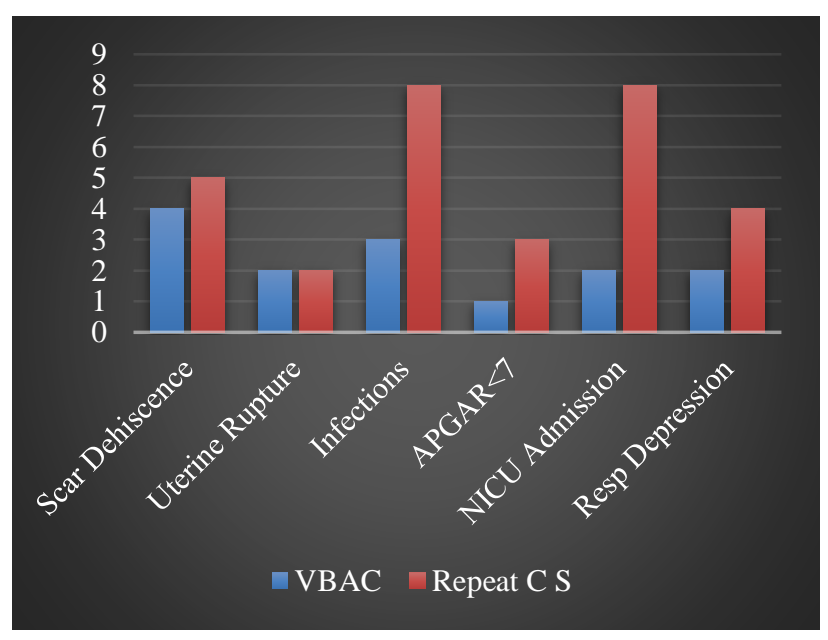

Figure 1: Complications in subjects.

\section{DISCUSSION}

The present study was conducted in a tertiary care hospital and teaching institution in Udaipur Rajasthan. The study had a sample pool of 30 subjects who consented to undergo TOLAC among whom $46.7 \%$ subjects underwent VBAC successfully while rest had to be opted for emergency LSCS owing to indications or maternal request. In studies by Landon B et al and Stone et al the rate of VBAC success was between 56 to $77 \%$ which is extensively higher than our reported outcome rate. ${ }^{6,7}$ This is not in concurrence with our findings. The rate of $47.9 \%$ was reported in an Indian study by Prabha et al which is similar to our findings. The reasons for the higher VBAC success rates in the studies by western authors can be attributed to the fact that the ANC care was same but maternal opt out was lower than in our study. ${ }^{8}$ An African study comprised analytical data from 14 different clinical trial scenarios and reported that the average VBAC success rate was $69 \%$ in the studies. This is again on the higher side as compared to our sample. 
We had no incidence of maternal or neonatal mortality in our study sample, but it has been reported in various studies that maternal mortality rate of 1.9 per lakh has been reported when TOLAC has been attempted. This is still lower than a reported mortality rate of 9.6 per lakh for repeated cesarian cases. ${ }^{9}$ Our study had a incidence of scar dehiscence as commonest complication at $13.34 \%$. Compared to Landon et al the incidence is higher in our study sample. ${ }^{7}$ In the study group, the incidence of postdelivery infection was higher in repeated $\mathrm{C}$ section group as compared to the VBAC group. This is in conformity with the studies by Guise JM et al who reported that various studies from different parts of the world reported a similar trend with upto $19 \%$ of all repeat cesarian cases being prone and diagnosed with infective pathologies. ${ }^{10}$ Neonatal outcomes also showed a significant difference in between the two groups with adverse outcomes being higher in the cesarian group. This is in concordance with studies by Fisler RE et al and Kamath BD et al wherein the authors also reported a higher incidence of unfavorable outcomes in repeat cesarian groups. ${ }^{9,11}$ However, an Indian study by Prabha et al stated a different complication outcome wherein the authors reported no statistically significant difference between the two groups. ${ }^{8}$

\section{CONCLUSION}

Despite having a lower VBAC success rate the study did report that it is possible and significantly better in terms of prognosis for Indian women to undergo TOLAC and VBAC with satisfactory neonatal outcomes and low maternal complications. The study revealed that the outcomes were on the lower side but possible, a larger sample size or more diverse obstetric population may show a trend of results which are in accordance with the reports of western authors. The study was limited by the small sample size and lack of a long term follow up to ascertain the complication in later stages if any. The study did conclude that despite some studies not in concurrence, the VBAC is safe and effective delivery modality in the population studied. Further large scale studies may be needed to further cement this hypothesis. The major limitation in the study is the low sample size. The larger sample size spread over a longer duration may provide a better representative idea of the actual objective behind this study.

\section{Funding: No funding sources}

Conflict of interest: None declared

Ethical approval: The study was approved by the Institutional Ethics Committee

\section{REFERENCES}

1. Viswanathan M, Visco AG, Hartmann K, Wechter ME, Gartlehner G, Wu JM, et al. Cesarean delivery on maternal request. Evidence report/technology assessment. 2006;(133):1.

2. National Institutes of Health Consensus Development Conference Panel. National Institutes of Health Consensus Development conference statement: vaginal birth after cesarean: new insights March 8-10, 2010. Obstet Gynecol. 2010;115(6):1279-95.

3. Hamilton B, Martin J, Ventura S. Births: Preliminary Data for 2009. National Statistics Reports. Vol 59. Hyattsville, MD: National Center for Health Statistics; 2010.

4. Cahill AG, Stamilio DM, Odibo AO, Peipert JF, Ratcliffe SJ, Stevens EJ, et al. Is vaginal birth after cesarean (VBAC) or elective repeat cesarean safer in women with a prior vaginal delivery? Am J Obstet Gynecol. 2006;195:1143-7.

5. Grobman WA, Lai Y, Landon MB, Spong CY, Leveno KJ, Rouse DJ, et al. Can a prediction model for vaginal birth after cesarean also predict the probabilit y of morbidity related to a trial of labor? Am J Obstet Gynecol. 2009;200:56.e1-6.

6. Landon MB, Hauth JC, Leveno KJ, Spong CY, Leindecker S, Varner $\mathrm{W}$, et al. Maternal and perinatal outcomes associated with trial of labour after prior caesarean delivery. NEJM. 2004;351:2581-9.

7. Stone C, Halliday J, Lumley J, Brennecke S. Vaginal birth after caesarean (VBAC): a population study. Paediatr Perinat Epidemiol. 2000;14:340-8.

8. Prabha S, Naralkar M, Prema. Outcome of post cesarean pregnancy in a tertiary care centre in south India. The New Indian Journal of Obgyn. 2020;7(1): 31-5.

9. Fisler RE, Cohen AC, Ringer SA, Lieberman E. Neonatal outcome after trial of labor compared with elective repeat cesarean section. Birth. 2003;30(2): 83-8.

10. Guise JM, Eden K, Emeis C, Denman M, Marshall $\mathrm{N}, \mathrm{Fu} \mathrm{R}$, et al. Vaginal birth after cesarean: new insights. Evidence report/technology assessment no.191. AHRQ Publication No. 10-E003. Rockville (MD): Agency for Healthcare Research and Quality; 2010.

11. Kamath BD, Todd JK, Glazner JE, Lezotte D, Lynch AM. Neonatal outcomes after elective cesarean delivery. Obstet Gynecol. 2009;133(6):1231-8.

Cite this article as: Sharma $\mathrm{A}$, Jadeja $\mathrm{H}$, Malhotra $\mathrm{P}$. Outcome of post caesarian pregnancy in a tertiary care hospital in Udaipur, Rajasthan. Int J Reprod Contracept Obstet Gynecol 2021;10:1614-6. 